Marquette University

e-Publications@Marquette

Biomedical Engineering Faculty Research and

Publications

Biomedical Engineering, Department of

$1-1-2012$

\title{
Stroke-related Changes in Neuromuscular Fatigue of the Hip Flexors and Functional Implications
}

Allison Hyngstrom

Marquette University

Tanya Onushko

Marquette University

Robert Heitz

Marquette University, robert.heitz@marquette.edu

Anthony Rutkowski

Georgia Institute of Technology - Main Campus

Sandra K. Hunter

Marquette University, sandra.hunter@marquette.edu

See next page for additional authors

Accepted version. American Journal of Physical Medicine \& Rehabilitation, Vol.91, No. 1, (January

2012): 33-42. DOI. (C) 2012 Lippincott Williams \& Wilkins, Inc. Used with permission. 
Authors

Allison Hyngstrom, Tanya Onushko, Robert Heitz, Anthony Rutkowski, Sandra K. Hunter, and Brian D. Schmit 


\title{
Stroke-related Changes in Neuromuscular Fatigue of the Hip Flexors and Functional Implications
}

\author{
Allison S. Hyngstrom, PhD \\ Department of Physical Therapy, Marquette University \\ Milwaukee, WI
}

Tanya Onushko, MS

Department of Biomedical Engineering, Marquette University

Milwaukee, WI

Robert P. Heitz, MS

Department of Biomedical Engineering, Marquette University

Milwaukee, WI

Anthony Rutkowski, BS

Department of Physical Therapy, Marquette University

Milwaukee, WI

Sandra K. Hunter, PhD

Department of Physical Therapy, Marquette University

Milwaukee, WI

Brian D. Schmit, PhD

Department of Biomedical Engineering, Marquette University

Milwaukee, WI

American Journal of Physical Medicine and Rehabilitation, Vol. 91, No. 1 (January 2012): pg. 33-42. DOI. This article is (C) Lippincott Williams \& Wilkins, Inc. and permission has been granted for this version to appear in e-

Publications@Marquette. Lippincott Williams \& Wilkins, Inc. does not grant permission for this article to be further

copied/distributed or hosted elsewhere without the express permission from Lippincott Williams \& Wilkins, Inc.. 
NOT THE PUBLISHED VERSION; this is the author's final, peer-reviewed manuscript. The published version may be accessed by following the link in the citation at the bottom of the page.

\section{Abstract: \\ Objective}

To compare stroke-related changes in hip-flexor neuromuscular fatigue of the paretic leg during a sustained, isometric sub-maximal contraction with the non-paretic leg and controls, and correlate fatigue with clinical measures of function.

\section{Design}

Hip torques were measured during a fatiguing hip-flexion contraction at $20 \%$ of the hip flexion maximal voluntary contraction (MVC) in the paretic and nonparetic legs of 13 people with chronic stroke and 10 age-matched controls. In addition, participants with stroke performed a fatiguing contraction of the paretic leg at the absolute torque equivalent to $20 \%$ MVC of the non-paretic leg and were tested for self-selected walking speed (Ten-Meter Walk Test) and balance (Berg).

\section{Results}

When matching the non-paretic target torque, the paretic hip flexors had a shorter time to task failure compared with the non-paretic leg and controls $(p<0.05)$. Time to failure of the paretic leg was inversely correlated with the reduction of hip flexion MVC torque. Self-selected walking speed was correlated with declines in torque and steadiness. Berg-Balance scores were inversely correlated with the force fluctuation amplitude.

\section{Conclusions}

Fatigue and precision of contraction are correlated with walking function and balance post stroke.

Keywords: Neuromuscular Fatigue, Hip Flexors, Stroke.

\section{INTRODUCTION}

Stroke-related muscle fatigue, particularly of the hip flexors, may interfere with functional mobility and contribute to long term disability. Many motor tasks that are challenging to stroke survivors require sub-maximal symmetric torque production in bilateral hip joints to optimize postural stability and energy efficiency. For example during abnormal center of mass in standing leading to abnormal balance strategies or asymmetries in kinematics related to decreased 
power of the paretic leg 1 . Weakness of the paretic hip certainly contributes to functional mobility ${ }^{2}-4$; specifically it has been associated with decreased gait velocity. Muscle weakness post stroke has been attributed to muscle atrophy and also impaired voluntary activation because the nervous system is not able to adequately activate the available muscle $\underline{\underline{5}}-\underline{8}$. Functionally, asymmetric leg kinematics and kinetics have been associated with loss of power (i.e. weakness) and coordination in the paretic hip during walking $\underline{3}, \underline{9}$. The implications of this are that people with stroke have difficulty walking with efficiency over time.

While weakness is clearly an important factor that contributes to walking impairments, an increased susceptibility to fatigue also likely compounds baseline difficulties in generating hip torque. For example, people with stroke demonstrate alterations in kinematics after short bouts of walking $\underline{10}-\underline{12}$ and decreased distance walked during the 6minute walk test compared with healthy controls $\frac{13}{}$. While all of the mechanisms underlying these impairments in walking kinematics and distance walked are not fully understood, neuromuscular fatigue in the paretic hip likely limits motor function by exacerbating strength limitations. Although output is limited at the muscle, factors upstream of the neuromuscular junction (ie central) could play a role as well 14 .

The contribution of stroke-related neuromuscular fatigue to limitations in functional walking has been largely untested. Neuromuscular fatigue, defined as an exercise-induced reduction in force or power, can be quantified as the reduction in strength over time or as the duration of sub-maximal sustained tasks $\underline{14}, \underline{15}$. Although stroke patients frequently self-report generalized or systemic fatigue ${ }^{16}$, the examination of stroke-related neuromuscular fatigue has been limited and no studies have addressed changes in the hip flexor muscles.

Fatigue of the paretic hip flexors, in comparison to other leg muscles, is likely to greatly impact walking function post-stroke. Evidence in both human and animal models indicates an important role of hip musculature in the execution of leg movements and the control of the whole leg during walking $\frac{17-19}{19}$. Thus, changes in neuromuscular fatigue of the hip flexors in people post-stroke could not only limit walking distance and speed but may also interfere with the volitional 
control of joints distal to the hip. Moreover, because ankle propulsion by the plantar flexors can be significantly compromised post-stroke ${ }^{9}$, function of the hip flexors would become even more critical for limb advancement. Therefore, quantitative measures of neuromuscular fatigue of the paretic hip flexors are expected to be associated with walking function in people post-stroke.

The purpose of this study, therefore, was to 1) compare hip flexor neuromuscular fatigue using a sustained isometric sub-maximal contraction ${ }^{14}$ of the paretic and non-paretic limb in stroke patients and healthy controls, and 2) quantify neuromuscular fatigue of the paretic hip flexors and correlate fatigue metrics with tests of clinical function. We assessed the muscle fatigability of the paretic limb under two different conditions: (1) at $20 \%$ of maximal strength of the paretic and (2) $20 \%$ MVC of the non-paretic leg. The absolute torque condition was important because during gait and other bilateral leg tasks similar torque is required to produce symmetric movement patterns. Thus, constraining the paretic limb to generate a similar torque as the nonparetic leg during the fatiguing task could potentially expose changes in fatigability that contribute to motor impairment. We also measured maximal force development and variability as additional measures of performance that are affected by fatigue ${ }^{14}$. We hypothesized that (1)the paretic leg would exhibit a briefer time to task failure of the sub-maximal fatiguing contraction when the absolute target torque was matched with the non-paretic leg's target torque,(2) relative declines in maximal torque production would have stronger relationships with task failure than baseline levels of strength and (3) motor output variability of the paretic limb during the fatiguing task was expected to correlate more strongly with function compared with the non-paretic leg.

\section{SUBJECTS AND METHODS}

\section{Subjects}

Thirteen subjects with chronic post-stroke hemiparesis were recruited for this study (Table 1 ). Chronic stroke is defined here as six months post event. Subjects reported a single cortical stroke incident with subsequent unilateral impairment in sensory and/or motor function. All but one subject (S11) reported injury in the MCA territory. Nine of the 
thirteen participants were able to independently ambulate on even surfaces without the use of an orthotic or assistive device. Four subjects, (S1, S3, S4 and S10) utilized an ankle-foot orthosis and S4 and S10 ambulated with a single point cane. All subjects reported they did not use a wheelchair. Participants were excluded if they reported disturbances in vision or neglect, any untreated cardiovascular or orthopedic conditions, osteoporosis, decubitis ulcerations, or if cognitive limitations impaired their ability to adhere to instructions or provide informed consent. Ten age matched controls (mean age = 59.4 yrs old \pm 11.2 , 5 male; 5 female, mean \pm stdev Physical Activity Questionairre $^{20}=15.86 \pm 11$ Met hr/week ) with no history of neurological injury also participated in the study.

\begin{tabular}{|c|c|c|c|c|c|c|c|c|c|}
\hline Stroke & Age & Sex & Classification & $\begin{array}{l}\text { Hemisphere } \\
\text { Affected }\end{array}$ & $\begin{array}{l}\text { Time Since Stroke } \\
\text { (months) }\end{array}$ & $\begin{array}{c}\text { Fugl Meyer (Lower } \\
\text { Extremity) }\end{array}$ & $\begin{array}{l}\text { Walking Speed } \\
\qquad(\mathrm{m} / \mathrm{s})\end{array}$ & $\begin{array}{c}\text { Berg Balance } \\
\text { Test }\end{array}$ & $\begin{array}{c}\text { P.A.Q. } \\
\text { Met-Hr/wk }\end{array}$ \\
\hline $\mathrm{S} 1^{*}$ & 46 & M & Ischemic & $\mathrm{R}$ & 89 & 22 & 1.17 & 51 & 24.7 \\
\hline S2 & 58 & $\mathrm{~F}$ & Ishcemic & $\mathrm{R}$ & 240 & 32 & 1.27 & 49 & 21.8 \\
\hline $\mathrm{S} 3^{*}$ & 62 & $\mathrm{~F}$ & Ischemic & L & 64 & 25 & 0.38 & 20 & 9.7 \\
\hline $\mathrm{S} 4-^{*}$ & 45 & M & Ischemic & L & 58 & 22 & 0.70 & 33 & 10 \\
\hline S5 & 61 & $\mathrm{~F}$ & Ischemic & L & 94 & 32 & 1.30 & 54 & 14.3 \\
\hline S6 & 52 & $\mathrm{~F}$ & Ischemic & $\mathrm{R}$ & 204 & 31 & 1.27 & 56 & 22 \\
\hline S7 ${ }^{*}$ & 51 & $\mathrm{M}$ & Ischemic & L & 40 & 24 & 1.15 & 46 & 11.4 \\
\hline S8- & 62 & $\mathrm{M}$ & Ischemic & R & 51 & 27 & 1.24 & 49 & 14.7 \\
\hline $\mathrm{S} 9{ }^{*}$ & 60 & $\mathrm{~F}$ & Hemorrhagic & L & 296 & 19 & 0.84 & 46 & 13.6 \\
\hline $\mathrm{S} 10$ & 48 & M & Hemorrhagic & L & 84 & 24 & 0.74 & 45 & 9.9 \\
\hline S11- & 54 & M & Ischemic & $\mathrm{R}$ & 54 & 32 & 1.54 & 54 & 32.0 \\
\hline $\mathrm{S} 12$ & 58 & $\mathrm{~F}$ & Ischemic & L & 67 & 23 & 0.63 & 38 & 0.34 \\
\hline $\mathrm{S} 13$ & 57 & M & Ischemic & $\mathrm{R}$ & 96 & 29 & 1.36 & 46 & 16.99 \\
\hline
\end{tabular}

TABLE 1 Characteristics of Stroke Subjects

*Subject participated in both equal effort and equal torque conditions

P.A.Q. = Physical Activity Questionnaire

Prior to the fatigue testing, participants with stroke underwent a clinical evaluation that included: passive and active range of motion of bilateral lower extremities, the lower extremity Fugl-Meyer test (ability to fractionate lower extremity joint movement), the Berg Balance Test (examines both static and dynamic sitting and standing balance), and the Ten Meter Walk (quantifies self selected walking speed). All evaluations were done by the same licensed physical therapist.

American Journal of Physical Medicine and Rehabilitation, Vol. 91, No. 1 (January 2012): pg. 33-42. DOI. This article is (C) Lippincott Williams \& Wilkins, Inc. and permission has been granted for this version to appear in e- 
Subjects were given 5 minutes rest between assessments and at least 15 minutes break before the fatigue testing begun. 15 minutes is adequate to recover from peripheral fatigue and central fatigue resolves within a few minutes $\underline{14}$. In addition, a criteria to begin testing was the return of blood pressure and heart to resting values.

Seven of the thirteen participants with stroke recruited in this study (refer to Table 1) also participated in a second testing session on a separate day (at least one week apart). All individual with stroke were invited to return for a second session, but some participants could not because of scheduling conflicts. Informed consent was obtained from both stroke and control participants and all experimental procedures were approved by the Institutional Review Board of Marquette University.

\section{Test Apparatus and Set-up}

Subjects were assisted onto a height adjustable therapy plinth and into the robotic test apparatus used to obtain all lower extremity torque measurements. The apparatus consisted of two servomotors (Kollmorgen, Northampton, MA) and adjoining leg braces instrumented with torque transducers (S. Himmelstein and Company, Hoffman Estates, IL). For each leg, the head of the greater trochanter was aligned with the axis of rotation of the servomotors. Likewise, the anatomical center of rotation for the ankles and knees were aligned with the center axis of torque transducers within the leg braces. Sagittal plane torques of the hips were measured during MVC measurements and a sub-maximal fatiguing task. Optical encoders (US Digital, Vancouver, WA) monitored hip position. Throughout all testing protocols bilateral knees and ankles were held static at $20^{\circ}$ flexion and $90^{\circ}$ plantarflexion respectively.

Prior to acquisition, torque signals were low-pass filtered (500 $\mathrm{Hz}$ ) and then sampled at $1 \mathrm{kHz}$ using a data acquisition card (National Instruments Corp., Austin, TX) and PC. Custom-written LabVIEW (National Instruments Corp.) programs were used to generate a hip trajectory command signal to the servocontroller and acquire all data. 
NOT THE PUBLISHED VERSION; this is the author's final, peer-reviewed manuscript. The published version may be accessed by following the link in the citation at the bottom of the page.

\section{Experimental Design and Protocol}

This was an observational, case-controlled study. Subjects were familiarized with the robotic device and allowed to practice brief MVC and sustained contraction protocols prior to experimental testing. All subjects first performed 3-5 isometric hip flexion MVCs for each leg. The test leg was positioned at $20^{\circ}$ hip flexion and the contralateral hip at $0-5^{\circ} \mathrm{s}$ hip extension. MVC trials were repeated until peak forces from two of the three trials were $<5 \%$ of each other. One minute breaks were given between MVCs. Visual feedback was displayed on a computer monitor and subjects were verbally encouraged throughout each MVC trial. A ten minute rest was given between MVCs and the fatiguing protocol.

Next, for the fatiguing protocol, subjects performed unilateral, sustained sub-maximal isometric hip flexor muscle contractions. During a given testing session, participants with stroke performed one of the following two protocols:

1. Equal Effort (EE): Subjects performed a sub-maximal contraction matching $20 \%$ of the hip flexion torque MVC of the same leg being tested (e.g. the target torque during the sustained contraction of paretic leg testing was calculated from the paretic leg MVC). For the remainder of this manuscript, we will refer to this torque measurement as the "ipsilateral hip flexion torque."

2. Equal Torque (ET): The target torques used during the paretic and non-paretic sustained contractions were $20 \%$ of the nonparetic hip flexion MVC (i.e. the paretic target torque was the same as the non-paretic target torque).

Control subjects performed sustained sub-maximal isometric hip flexor muscle contractions of their dominant leg with target torques that were $20 \%$ of the MVC of the leg being tested. Task failure was defined as an inability to maintain the target torque within a $\pm 10 \%$ window for $\geq 4 \mathrm{~s}$ or $\geq 3$ failures within a 5 s continuous window. Subjects were instructed to continue with the task until instructed to stop. Immediately following task failure, subjects performed a final hip flexor MVC. For participants with stroke, after a rest period of 10-15

American Journal of Physical Medicine and Rehabilitation, Vol. 91, No. 1 (January 2012): pg. 33-42. DOI. This article is (C) Lippincott Williams \& Wilkins, Inc. and permission has been granted for this version to appear in e- 
NOT THE PUBLISHED VERSION; this is the author's final, peer-reviewed manuscript. The published version may be accessed by following the link in the citation at the bottom of the page.

minutes, the sustained sub-maximal contraction was repeated for the opposite hip. Order of paretic leg versus non-paretic hip testing was counterbalanced. Joint torques from the hipswere low-pass filtered at $5 \mathrm{~Hz}$, and the torque contribution from gravity of the leg and leg brace were subtracted from the data prior to further analysis $\underline{21}$.

\section{Data Analysis}

Pre-fatigue MVC Measurements The hip flexor torque MVC prior to the fatiguing task (pre-MVC) was the maximum hip torque value across the initial MVC trials. The rate and time-to-peak contraction were calculated for the pre MVC torque traces. Using the derivative of the MVC torque trace, the rate of contraction was calculated by finding the maximum slope during the time of contraction. The time-to-peak contraction was considered to be the time between the onset of contraction and the peak torque. The onset of the contraction was determined by finding the time of the minimum value of the derivative of the MVC torque trace. The measurements above were made to determine baseline levels of strength (Pre-MVC) necessary to determine the magnitude of fatigue post fatigueing protocol and to assist with global categorization of motor unit recruitment during an MVC (rate of contraction and time to peak contraction).

\section{Sustained Sub-Maximal Fatiguing Contraction Measurements:} Time to Task Failure Time to task failure (seconds) was determined by the failure criteria described above (please see Protocol subsection). How long an individual can perform a sub-maximal task is also a metric of fatigue that can be independent of decreases in MVC 14 . In other words, a person can have decreases in MVC, but still be able to continue with a sub-maximal task.

\section{Sustained Sub-Maximal Fatiguing Contraction: Force Fluctuation}

Measurements Force fluctuations or variability are a metric of fatigue and the relative magnitude of the force fluctuations is a metric of steadiness or control that can worsen or decline with fatigue. In order to determine the relative magnitude of force fluctuations, data from the fatiguing task were divided into ten equal segments - the entire hip flexion torque measurement was split into $10 \%$ segments of the total duration. For each $10 \%$ segment, fluctuations in motor output were quantified as the coefficient of variation (CV) of the hip flexion

American Journal of Physical Medicine and Rehabilitation, Vol. 91, No. 1 (January 2012): pg. 33-42. DOI. This article is (C) Lippincott Williams \& Wilkins, Inc. and permission has been granted for this version to appear in e-

Publications@Marquette. Lippincott Williams \& Wilkins, Inc. does not grant permission for this article to be further copied/distributed or hosted elsewhere without the express permission from Lippincott Williams \& Wilkins, Inc.. 
NOT THE PUBLISHED VERSION; this is the author's final, peer-reviewed manuscript. The published version may be accessed by following the link in the citation at the bottom of the page.

torque (standard deviation/mean torque) for the paretic, non-paretic, and control legs.

Post-fatigue MVC Measurements Ultimately, reductions in force following the sub-maximal contraction demonstrate neuromuscular fatigue. As with the determination of the pre-fatigue MVC, the maximum hip flexor torque following the fatiguing contraction was the post-MVC value. The ratio of MVC decline (post-MVC/pre-MVC) was used to normalize for baseline strength for the correlation with time to task failure. In other words, the ratio of MVC decline was used to illustrate that baseline strength was not related with time of task failure The difference between the hip flexor MVC before and after the fatiguing contraction was calculated $(\triangle M V C)$ in order to relate the absolute magnitude of neuromuscular fatigue with measures of clinical function.

\section{Statistical Analysis}

Data are reported as means \pm standard deviation. Paired student t-tests were used to detect significant differences between the paretic and non-paretic leg mean MVC amplitudes ( $a=0.05)$. Separate one-way analysis of variance (ANOVAs) for each condition (EE) and (ET) were used to detect significant differences between the mean duration for each sub-maximal sustained contraction between stroke (paretic and non-paretic legs) and control subjects $(a=0.05)$. A Tukey post-hoc analysis was used for pairwise comparisons. The Pearson product-moment correlation coefficient $\left(r^{2}\right)$ was used to determine associations between variables. To establish if associations were present, separate correlations were performed between the time to task failure and the following variables (respectively): pre-fatigue MVC, pre-fatigue MVC (paretic/non-paretic), and ratio of MVC decline. Associations were also done between metrics of fatigue and clinical measures of function. Walking speed (calculated from the 10 meter walk test) was separately correlated with the following variables: the absolute decline in paretic hip MVC, and paretic C.V. Likewise, the Berg Balance Score was separately correlated with the following variables: the absolute decline in paretic hip MVC, and paretic C.V. 
NOT THE PUBLISHED VERSION; this is the author's final, peer-reviewed manuscript. The published version may be accessed by following the link in the citation at the bottom of the page.

\section{RESULTS}

\section{Clinical Assessments}

As evaluated by a licensed physical therapist, no participant had ROM restrictions that prohibited positioning within the testing apparatus. The following subjects exhibited velocity dependent resistance to stretch in one or more muscles of their affected leg: S1, S3, S4, S7, S8, S9, S10, and S12. The mean lower extremity Fugl Meyer score was $26 \pm 5$ (Table 1). On average, the participants with stroke had a self-selected walking speed of $1.03 \mathrm{~m} / \mathrm{s} \pm 0.35$ (Table 1 ). Although $6 / 13$ had walking speeds consistent with normative values $(1.2 \mathrm{~m} / \mathrm{s})^{1}$, all subjects, by observation, demonstrated asymmetric walking kinematics between the paretic and non-paretic legs and $7 / 13$ subjects had impaired walking speed. For the Berg Balance Test, the participants with stroke had a mean score of $44 \pm 10$ (Table 1 ).

\section{Pre-fatigue MVC Measurements}

The mean paretic hip flexor MVC measured before the sustained fatiguing contraction was smaller compared with the non-paretic hip flexor MVC for both protocols (Table 2, paired t-test, $p<0.05$, see Fig. 1 for single subject example). The paretic leg pre-fatigue MVC was characterized by a slower rate of torque development $(350 \mathrm{Nm} / \mathrm{s} \pm$ $230, p<0.025)$ compared with the non-paretic leg $(500 \pm 330 \mathrm{Nm} / \mathrm{s})$ and controls $(660 \mathrm{Nm} / \mathrm{s} \pm 340)$ and a longer time to peak contraction $(3.0 \mathrm{~s} \pm 1.8, \mathrm{p}<0.025)$ compared with the non-paretic leg $(2.0 \mathrm{~s} \pm$ $0.9)$ and controls (1.4 $\mathrm{s} \pm 0.5)$.

American Journal of Physical Medicine and Rehabilitation, Vol. 91, No. 1 (January 2012): pg. 33-42. DOI. This article is C Lippincott Williams \& Wilkins, Inc. and permission has been granted for this version to appear in ePublications@Marquette. Lippincott Williams \& Wilkins, Inc. does not grant permission for this article to be further copied/distributed or hosted elsewhere without the express permission from Lippincott Williams \& Wilkins, Inc.. 
A


Paretic
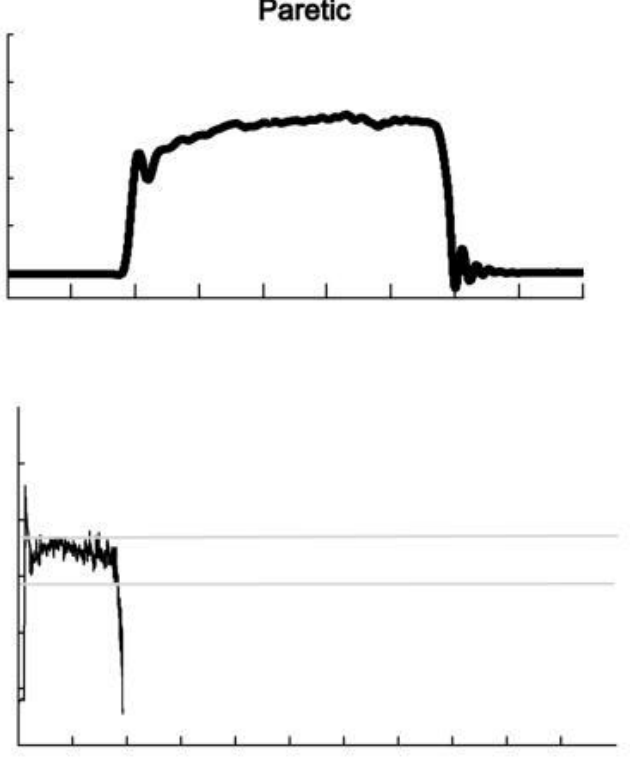

Figure 1 Single subject data for pre and post MVC measurements and fatiguing contractions. (A) Torque generated during a maximal voluntary contraction (MVC) for the non-paretic and paretic leg (S11). (B) Torque generated during the sub-maximal contraction trial for the (ET) protocol (S8).

\begin{tabular}{lccccc}
\hline & \multicolumn{2}{c}{ Equal Effort } & \multicolumn{2}{c}{ Equal Torque } & Control \\
\hline & Paretic & Non-paretic & Paretic & Non-paretic & Dominant Leg \\
\cline { 2 - 6 } & & & & \\
MVC Pre (Nm) & $83 \pm 37$ & $101 \pm 54$ & $78 \pm 27$ & $96 \pm 29$ & $108 \pm 32$ \\
Post MVC (Nm) & $48 \pm 24$ & $57 \pm 26$ & $43 \pm 22$ & $49 \pm 19$ & $76 \pm 32$ \\
$\%$ Decline & $42 \pm 18$ & $38 \pm 21$ & $46 \pm 22$ & $48 \pm 13$ & $31 \pm 17$ \\
\hline
\end{tabular}

TABLE 2 MVC Measurements

\section{Time to Task Failure}

During the EE protocol, the paretic and non-paretic leg generated a target hip flexor torque of $20 \%$ of the ipsilateral MVC. The paretic leg's time to task failure was not significantly different than the non-paretic leg and control leg (Fig. 2A: paretic $=318 \pm 204 \mathrm{~s}$ vs. non-paretic $=425 \pm 511 \mathrm{~s}$ vs. control $=399 \pm 249 \mathrm{~s}, \mathrm{df}=29, \mathrm{~F}=$ 
$.253, p=0.779)$. For the ET protocol, the paretic leg maintained a target torque that was $20 \%$ of the non-paretic leg's hip flexor MVC. On average, this resulted in a target torque that was $26 \%$ of the paretic leg's MVC (a larger absolute torque magnitude compared with EE). For this sustained contraction, the paretic leg had a significantly shorter time to task failure compared with the non-paretic and control legs (Fig. $2 \mathrm{~B}$, paretic $=126 \pm 144 \mathrm{~s}$ vs. non-paretic $382 \pm 242 \mathrm{~s}$ vs. control $397 \pm 249 \mathrm{~s}, \mathrm{df}=28, \mathrm{~F}=4.865, \mathrm{p}=0.016)$. For both protocols, the time to task failure of the paretic leg was not associated with either the pre-fatigue MVC of the paretic leg $\left(E E: r^{2}=0.03, E T: r^{2}=0.12\right)$ or the ratio of the paretic and non-paretic leg pre-fatigue MVC (EE: $r^{2}=$ $\left.0.007, \mathrm{ET}: r^{2}=0.06\right)$. Thus, the time to failure of the paretic leg was independent of the strength exerted and whether a participant with stroke was weaker as compared to the non-paretic side.
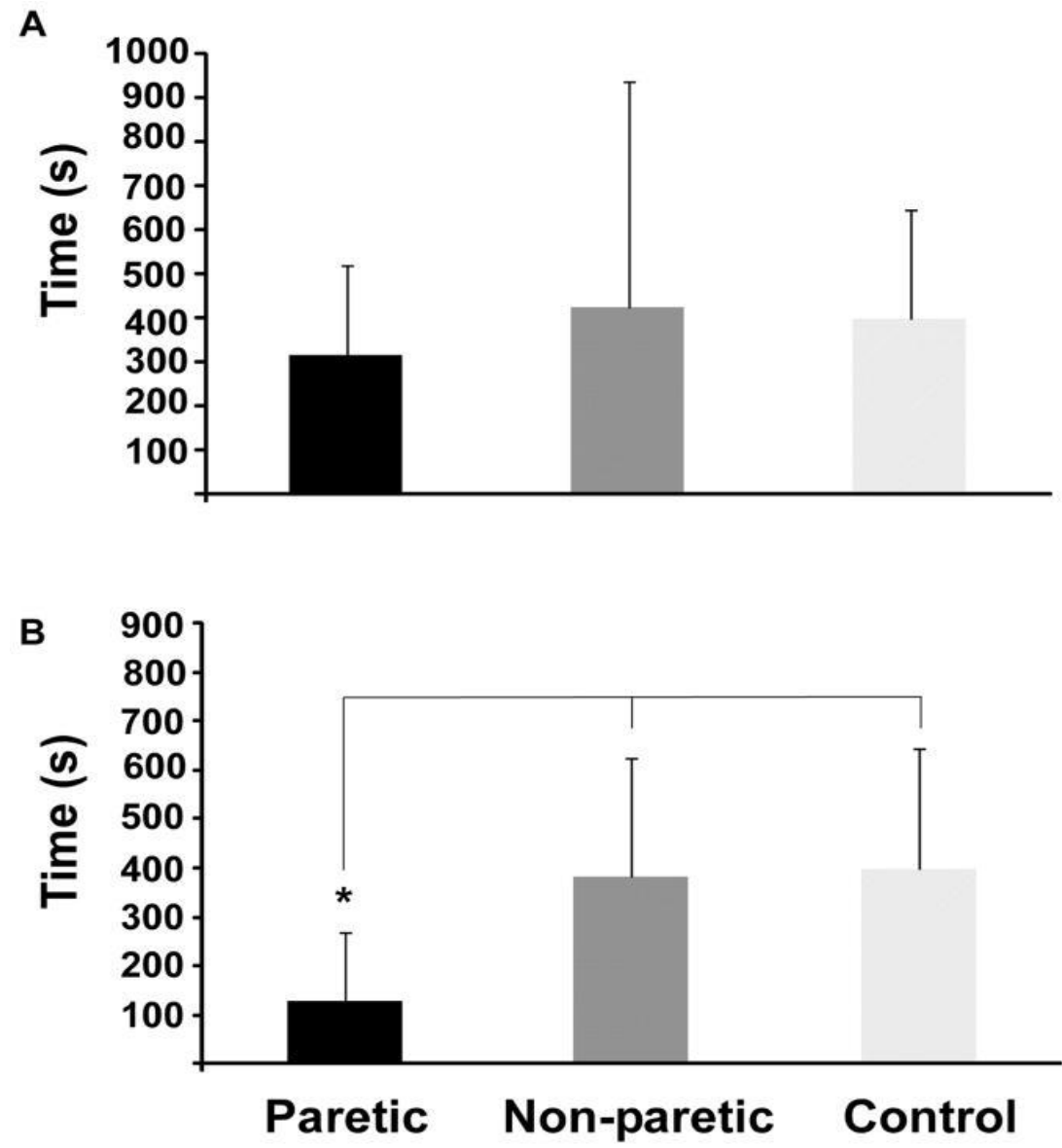

Figure 2 Mean duration of the sub-maximal hip flexion contraction for $(A)$ the equal effort protocol and (B) the equal torque protocol. Significance between groups is indicated by the asterisk. 


\section{Percent Decline in MVC Measurements}

There was a significant decline in MVC hip flexor torque after the fatiguing task for the paretic, non-paretic legs and control subjects (Table 2). Mean percent declines in MVC were not significantly different (ie no main effect of test leg group) between protocols (Table $\underline{2}$, ANOVA, df $=49, \mathrm{~F}=1.374, \mathrm{p}=0.25$ ).

\section{Ratio of MVC Decline}

For both protocols, however, the paretic leg's ratio of MVC decline (see Methods) was negatively correlated with the paretic leg's time to task failure (Fig. 3A; EE $r^{2}=0.42$, ET $r^{2}=0.34$ ). Participants with stroke with larger relative declines in MVC force following the sustained contraction had longer time to task failure. In contrast, there was no systematic relationship between the non-paretic and control leg's ratio of MVC decline and respective time to task failure (Fig. 3B, $r^{2} 0.2$ ) which is consistent with previous findings in healthy controls 22 .

A

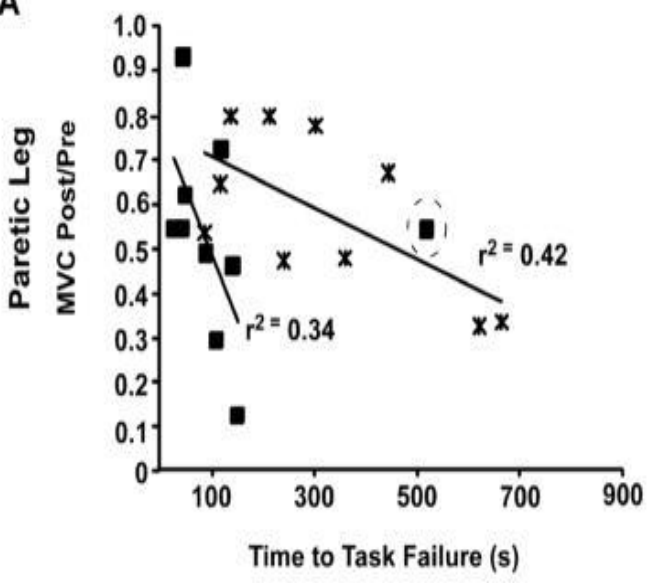

B

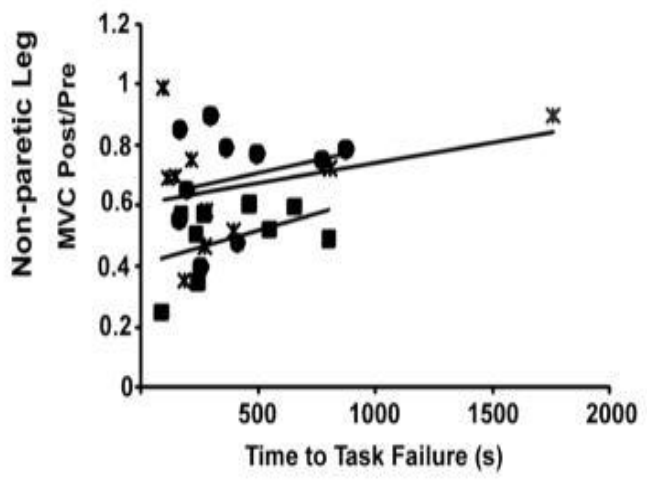

\section{XEqual Effort Protocol E Equal Torque Protocol - Control}

Figure 3 Correlations between the time to task failure duration with the Post/Pre MVC torque ratio for the $(A)$ paretic leg and $(B)$ the non-paretic leg and dominant leg of control subjects. The circled square in $(A)$ indicates an outlier not included in the correlation $(n=9)$. 


\section{Absolute Decline in Force Relates to Walking Speed}

The paretic leg's decline in hip flexor MVC torque for the ET protocol was positively correlated with self selected walking speed (Fig. 4A, $r^{2}=0.4$ ). That is, subjects with faster self selected walking speeds demonstrated a larger decline in MVC force following the sustained contraction when required to generate the same target torque as the non-paretic leg (ET condition). In contrast, there was no systematic relationship between the decline in paretic leg hip flexor MVC and walking speed for the EE protocol $\left(r^{2}<0.0009\right)$ or the nonparetic leg for both protocols (EE: $\left.r^{2}=0.01 ; E T: r^{2}=0.0012\right)$. The paretic decline in hip flexor MVC torque for the ET protocol was weakly positively correlated with the Berg Balance score, but the relationship $\left(r^{2}=0.26\right)$.

A

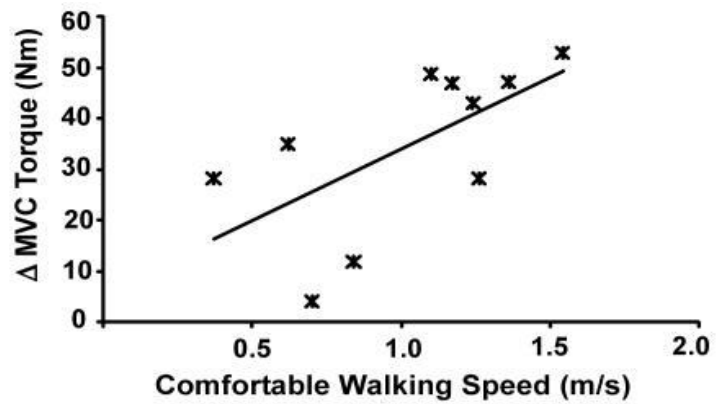

B

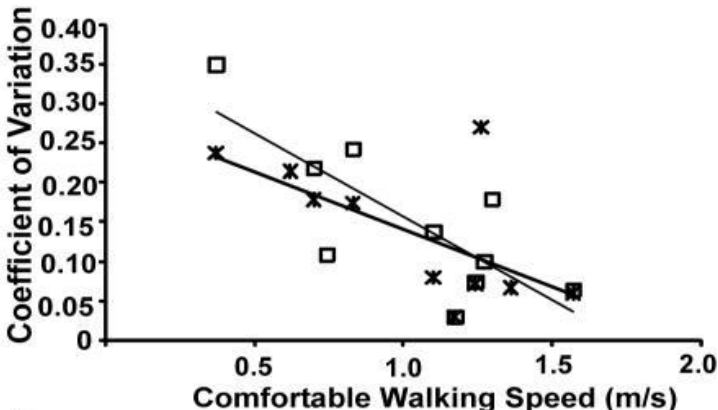

C

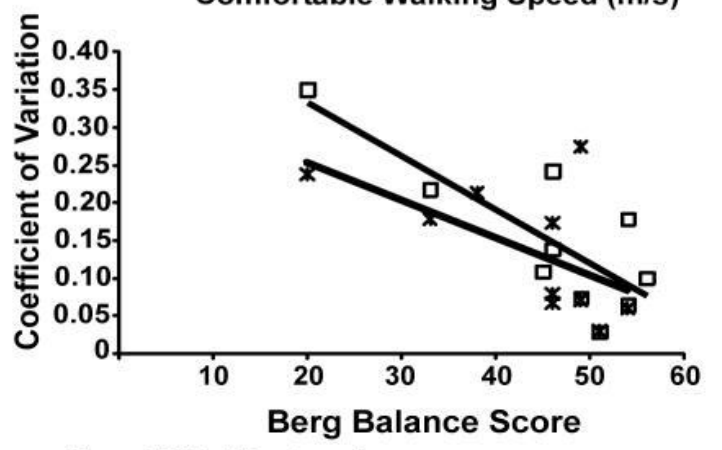

口 Equal Effort Protocol x Equal Torque Protocol

American Journal of Physical Medicine and Rehabilitation, Vol. 91, No. 1 (January 2012): pg. 33-42. DOI. This article is (C Lippincott Williams \& Wilkins, Inc. and permission has been granted for this version to appear in e-

Publications@Marquette. Lippincott Williams \& Wilkins, Inc. does not grant permission for this article to be further copied/distributed or hosted elsewhere without the express permission from Lippincott Williams \& Wilkins, Inc.. 
NOT THE PUBLISHED VERSION; this is the author's final, peer-reviewed manuscript. The published version may be accessed by following the link in the citation at the bottom of the page.

Figure 4 Correlations between functional measures and neuromuscular fatigue parameters. (A) The change in torque between pre-fatigue and post-fatigue MVC trials was positively correlated with comfortable walking speed in participants with stroke for the equal torque (ET) protocol. The coefficient of variation was negatively correlated with the Ten Meter Walk test (B) and Berg Balance Score (C) for both protocols.

\section{Coefficient of Variation and Measures of Walking Speed and Balance}

For both protocols, the mean CV (see Methods) calculated across the entire trial was not significantly different between the paretic ( $E E=0.15 \pm 0.09 ; E T=0.14 \pm 0.08)$, non-paretic leg ( $E E=$ $0.12 \pm 0.05 ; \mathrm{ET}=0.12 \pm 0.04)$ and control $(0.13 \pm 0.09)$ legs during the fatiguing contraction( $E E: d f=29, F=0.28, p=0.75$; $E T$ : $d f=29$, $\mathrm{F}=.09, \mathrm{p}=0.91)$. However, paretic leg CV negatively correlated with comfortable walking speed (Fig. 4B, EE: $r^{2}=0.6 ;$ ET: $r^{2}=0.41, p<$ 0.05 ) indicating that slower walkers had greater fluctuations in motor output. In addition, there was an inverse relationship between the paretic leg mean CV and the Berg Balance Test score (Fig. 4C, EE: $r^{2}=$ 0.64, $\left.\mathrm{p}<0.05 ; \mathrm{ET}: \mathrm{r}^{2}=0.35, \mathrm{p}<0.05\right)$.

\section{DISCUSSION}

The three key findings from this study are: 1) the paretic hip flexor muscles have a briefer time to task failure for sustained submaximal contractions compared with the non-paretic leg when absolute target torques were matched, 2) the time to task failure was inversely related to the paretic leg's decline in torque and 3 ) the magnitude of force fluctuations of the paretic limb during sustained hip flexion contractions was related to clinical measures of motor impairment and function. In addition to the well-documented findings that the paretic leg has deficits in maximal force production, we also demonstrated deficits of the paretic leg to sustain and control submaximal contractions compared with the non-paretic leg and healthy controls. These latter results are especially relevant because many activities of daily living, such as walking, involve sub-maximal contractions. This study exposes limitations in volitional control of sustained, sub-maximal tasks in the paretic leg - that is lower than to be expected given the small absolute differences in load as compared to the non-paretic leg 23

American Journal of Physical Medicine and Rehabilitation, Vol. 91, No. 1 (January 2012): pg. 33-42. DOI. This article is (C) Lippincott Williams \& Wilkins, Inc. and permission has been granted for this version to appear in e-

Publications@Marquette. Lippincott Williams \& Wilkins, Inc. does not grant permission for this article to be further copied/distributed or hosted elsewhere without the express permission from Lippincott Williams \& Wilkins, Inc.. 
In healthy adults, many studies support the role of both neural and muscular mechanisms contributing to the inability to maintain a contraction and fatigue $\underline{14}, \underline{15}, \underline{24}$. Our study suggests that neural (central) mechanisms that were independent of motivation may play a more dominant role (versus peripheral mechanisms) in the inability for participants with stroke to maintain a sub-maximal contraction as compared with the non-paretic and control legs. First, the time to task failure of the paretic leg was inversely correlated with the relative decrement in MVC torque of the paretic leg and this relationship was not evident for the non-paretic leg or control leg (Fig. 4). This indicates that the ability of the nervous system to sustain a steady drive to the muscle was the primary factor contributing to termination of the contractions. That is, participants with stroke who were not able to sustain the contractions (therefore demonstrated the briefest time to task failure) had the smallest decrements in MVC force. Thus, participants with stroke varied in their ability to sustain contractions in their paretic leg but the mechanism was not due to fatigue within the muscle, but rather an inability to sustain central drive that progressively increases during sustained contractions in healthy individuals $\underline{14}, \underline{25}$.

Second, the paretic muscle had a slower neuromuscular contraction compared with the non-paretic leg and control leg. Given that both the rate of peak contraction and the time to peak contraction were significantly slower suggests either a slower rate of recruitment of motor units due to weaker descending commands or a greater proportion of Type I fibers within the paretic muscle. If there was a stroke-related change in the proportion of fiber types only, the paretic leg would have exhibited a longer time to task failure, as seen in aging populations ${ }^{26}$. In contrast, the time to task failure of the paretic leg was less for the matched protocol and similar for the same relative intensity compared with the non-paretic leg. Alternatively, a slower rate of recruitment of hip flexor motor units in the paretic limb or an inability to recruit the available motor units would accelerate task failure possibly secondary to less efficient cycling of motor units that can occur during low-force sustained tasks $\underline{27}, \underline{28}$ Taken together, these results suggest although the nervous system can achieve desired submaximal torques at least during brief contractions post stroke, the strategy by which motor units are recruited may differ compared with the non-paretic leg or healthy controls. Functionally this may 
compromise the ability to sustain even a sub-maximal contraction or the ability of the paretic leg to perform repeated contractions.

Alternatively, participants with stroke may have terminated contractions as a function of motivation. Although we have no direct measurements of motivation, it is unlikely that lack of motivation contributed to a premature task failure of the paretic leg for several reasons. The subjects received continuous verbal and visual feedback in order to achieve maximal levels of external motivation.

Furthermore, the subjects did not stop the fatiguing contraction: rather the experimenter terminated the task based on criteria for task failure. Lastly within protocols, the average percent declines between the paretic and non-paretic leg MVCs were not significantly different demonstrating that the hip flexor muscles had reached similar physiological magnitudes of fatigue at task failure. Thus, that the participants with stroke were not able to sustain the matched torque of the paretic limb for reasons other than lack of motivation.

Our data also demonstrated that the precision of force control in the paretic limb could be associated with larger functional tasks related to walking. We showed that participants with stroke with larger force fluctuations during the fatiguing contraction had more impaired balance and were slower walkers (Fig. 5). Although previous studies have shown relationships between function and force fluctuations in healthy and aging adults $\underline{29}$ there has been limited study of force fluctuations in the stroke population. Loss of precision in sustaining a contraction may be due to either a loss of larger motor units resulting in a smaller recruitment range and therefore loss of resolution in control of motor output, more variable timing in motor unit activation or disturbance in the cortical visuomotor centers $\underline{22}, \underline{29}$.

Muscle fatigue is necessary for effective neuromuscular adaptation, increases in strength and rehabilitation. However, our study is the first to expose that the paretic muscle may terminate a sub-maximal contraction prior to the muscle being fully fatigued because of central deficits. Consequently, the inability to achieve larger magnitudes of muscle fatigue may prevent optimal neuromuscular adaptation and recovery in the paretic limb. Stroke patients, however, pose several challenges to achieving neuromuscular fatigue. For example, the magnitude of fatigue in the paretic leg can 
be influenced by substitution from other muscle groups and compensation from the non-paretic leg. Although compensatory movement strategies permit an individual to complete a motor task, they limit potential recovery by increasing the task's energetics. Furthermore, a loss of activity due to disability would negatively impact muscle endurance. Understanding the neural substrates that contribute to neuromuscular fatigue during a motor task will facilitate the development of targeted therapeutic interventions that optimize strength training post stroke.

Because, time to task failure can be improved with practice $\underline{30}, \underline{31}$ or altered with sensory input, training may improve a stroke patient's ability to sustain a contraction and heighten function. Although lowforce contractions are the foundation for many activities of daily living, neuromuscular fatigue can vary depending on the types of contractions (i.e isometric vs. concentric vs. eccentric). Limited by small sample size, future studies will address how the type of contraction affects stroke-related changes in fatigability and whether measures of fatigue from each type of contraction predict function post stroke. Additionally, in order to normalize neuromuscular fatigue post stroke it will be necessary to conduct experiments that provide information on the structures/pathways responsible for altered patterns of fatigue.

\section{Footnotes}

\section{Disclosures:}

Financial disclosure statements have been obtained, and no conflicts of interest have been reported by the authors or by any individuals in control of the content of this article. Supported by the American Heart Association: Clinical Research Program (10CRP2580011).

\section{References}

1. Perry J. Gait analysis. Thorofare: SLACK; 1992.

2. Nadeau S, Arsenault AB, Gravel D, Bourbonnais D. Analysis of the clinical factors determining natural and maximal gait speeds in adults with a stroke. Am J Phys Med Rehabil. 1999;78:123-130. 
NOT THE PUBLISHED VERSION; this is the author's final, peer-reviewed manuscript. The published version may be accessed by following the link in the citation at the bottom of the page.

3. De Quervain IA, Simon SR, Leurgans S, Pease WS, McAllister D. Gait pattern in the early recovery period after stroke. J Bone Joint Surg Am. 1996;78:1506-1514.

4. Hsu AL, Tang PF, Jan MH. Analysis of impairments influencing gait velocity and asymmetry of hemiplegic patients after mild to moderate stroke. Arch Phys Med Rehabil. 2003;84:1185-1193.

5. Swayne OB, Rothwell JC, Ward NS, Greenwood RJ. Stages of motor output reorganization after hemispheric stroke suggested by longitudinal studies of cortical physiology. Cereb Cortex. 2008;18:1909-1922.

6. Horstman AM, Beltman MJ, Gerrits KH, Koppe P, Janssen TW, Elich P, de Haan A. Intrinsic muscle strength and voluntary activation of both lower limbs and functional performance after stroke. Clin Physiol Funct Imaging. 2008;28:251-261.

7. Riley NA, Bilodeau M. Changes in upper limb joint torque patterns and emg signals with fatigue following a stroke. Disabil Rehabil. 2002;24:961969.

8. Patten C, Lexell J, Brown HE. Weakness and strength training in persons with poststroke hemiplegia: Rationale, method, and efficacy. J Rehabil Res Dev. 2004;41:293-312.

9. Jonkers I, Delp S, Patten C. Capacity to increase walking speed is limited by impaired hip and ankle power generation in lower functioning persons post-stroke. Gait Posture. 2009;29:129-137.

10. Dean $\mathrm{CM}$, Richards $\mathrm{CL}$, Malouin F. Walking speed over 10 metres overestimates locomotor capacity after stroke. Clin Rehabil. $2001 ; 15: 415-421$.

11. Bayat R, Barbeau H, Lamontagne A. Speed and temporal-distance adaptations during treadmill and overground walking following stroke. Neurorehabil Neural Repair. 2005;19:115-124.

12. Sibley KM, Tang A, Brooks D, McIlroy WE. Effects of extended effortful activity on spatio-temporal parameters of gait in individuals with stroke. Gait Posture. 2008;27:387-392.

13. Pohl PS, Duncan PW, Perera S, Liu W, Lai SM, Studenski S, Long J. Influence of stroke-related impairments on performance in 6-minute walk test. J Rehabil Res Dev. 2002;39:439-444.

American Journal of Physical Medicine and Rehabilitation, Vol. 91, No. 1 (January 2012): pg. 33-42. DOI. This article is (C) Lippincott Williams \& Wilkins, Inc. and permission has been granted for this version to appear in e-

Publications@Marquette. Lippincott Williams \& Wilkins, Inc. does not grant permission for this article to be further copied/distributed or hosted elsewhere without the express permission from Lippincott Williams \& Wilkins, Inc. 
NOT THE PUBLISHED VERSION; this is the author's final, peer-reviewed manuscript. The published version may be accessed by following the link in the citation at the bottom of the page.

14. Enoka RM, Duchateau J. Muscle fatigue: What, why and how it influences muscle function. J Physiol. 2008;586:11-23.

15. Gandevia SC. Spinal and supraspinal factors in human muscle fatigue. Physiol Rev. 2001;81:1725-1789.

16. Ingles JL, Eskes GA, Phillips SJ. Fatigue after stroke. Arch Phys Med Rehabil. 1999;80:173-178.

17. Grillner S, Rossignol S. On the initiation of the swing phase of locomotion in chronic spinal cats. Brain Res. 1978;146:269-277.

18. Hiebert GW, Whelan PJ, Prochazka A, Pearson KG. Contribution of hind limb flexor muscle afferents to the timing of phase transitions in the cat step cycle. J Neurophysiol. 1996;75:1126-1137.

19. Dean JC, Kuo AD. Elastic coupling of limb joints enables faster bipedal walking. J R Soc Interface. 2009;6:561-573.

20. Paffenbarger RS, Jr, Hyde RT, Hsieh CC, Wing AL. Physical activity, other life-style patterns, cardiovascular disease and longevity. Acta Med Scand Suppl. 1986;711:85-91.

21. Onushko T, Schmit BD. Reflex response to imposed bilateral hip oscillations in human spinal cord injury. J Neurophysiol. 2007;98:1849-1861.

22. Enoka RM, Christou EA, Hunter SK, Kornatz KW, Semmler JG, Taylor AM, Tracy BL. Mechanisms that contribute to differences in motor performance between young and old adults. J Electromyogr Kinesiol. 2003;13:1-12.

23. Frey Law LA, Avin KG. Endurance time is joint-specific: A modelling and meta-analysis investigation. Ergonomics. 2010;53:109-129.

24. Enoka RM, Stuart DG. Neurobiology of muscle fatigue. J Appl Physiol. $1992 ; 72: 1631-1648$.

25. Fuglevand $A J$, Zackowski KM, Huey KA, Enoka RM. Impairment of neuromuscular propagation during human fatiguing contractions at submaximal forces. J Physiol. 1993;460:549-572.

26. Narici M, Bordini M, Cerretelli P. Effect of age on human adductor pollicis muscle function. J Appl Physiol. 1991;71:1277-1281.

American Journal of Physical Medicine and Rehabilitation, Vol. 91, No. 1 (January 2012): pg. 33-42. DOI. This article is (C) Lippincott Williams \& Wilkins, Inc. and permission has been granted for this version to appear in e-

Publications@Marquette. Lippincott Williams \& Wilkins, Inc. does not grant permission for this article to be further copied/distributed or hosted elsewhere without the express permission from Lippincott Williams \& Wilkins, Inc.. 
NOT THE PUBLISHED VERSION; this is the author's final, peer-reviewed manuscript. The published version may be accessed by following the link in the citation at the bottom of the page.

27. Bawa $\mathrm{P}$, Murnaghan $\mathrm{C}$. Motor unit rotation in a variety of human muscles. J Neurophysiol. 2009;102:2265-2272.

28. Riley ZA, Maerz AH, Litsey JC, Enoka RM. Motor unit recruitment in human biceps brachii during sustained voluntary contractions. J Physiol. 2008;586:2183-2193.

29. Tracy BL. Advances in neuromuscular physiology of motor skills and muscle fatigue. Kerala: Research Signpost; 2009.

30. Hunter SK, Enoka RM. Changes in muscle activation can prolong the endurance time of a submaximal isometric contraction in humans. J Appl Physiol. 2003;94:108-118.

31. Riley ZA, Baudry S, Enoka RM. Reflex inhibition in human biceps brachii decreases with practice of a fatiguing contraction. J Neurophysiol. 2008; 100:2843-2851.

\section{About the Authors}

Allison S. Hyngstrom, PhD. : Department of Physical Therapy, Marquette University, P.O. Box 1881, Milwaukee, WI 53201-1881.

Phone: 414-288-4566, Fax: 414-288-7938

Email: allison.hyngstrom@marquette.edu

American Journal of Physical Medicine and Rehabilitation, Vol. 91, No. 1 (January 2012): pg. 33-42. DOI. This article is (C) Lippincott Williams \& Wilkins, Inc. and permission has been granted for this version to appear in e- 